\title{
Effect of tumor microenvironmental factors on the stability of tumor growth dynamics with nonzero correlation time
}

\begin{abstract}
The effect of tumor microenvironmental factors on tumor growth dynamics modeled by multiplicative colored noise is investigated. Using the Novikov theorem and Fox approach, an approximate Fokker--Planck equation for the non-Markovian stochastic equation is obtained and an analytic expression for the steady-state probability distribution Pst(x) is derived. We found that the strength of the microenvironmental factors $o$ have a negative effect on the stability of tumor growth at weak correlation time Üand at strong correlation time, the effect of $d$ is opposed and instead a positive growth stability is noticed for the tumor growth dynamics which in other words corresponds to growth effect. The result indicated that the growth effect exerted by the non-immunogenic components of tumor microenvironmental depend on the strength of the correlation time Ü
\end{abstract}

Keyword: Langevin equation; Fokker--Planck equation; Colored noise; Tumor growth dynamics 